\title{
Immunostimulatory and anti-neoplasm effects of a novel palindrome CpG oligodeoxynucleotide in mice
}

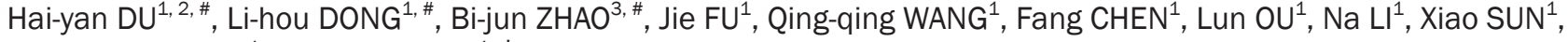 \\ Zhong-ming TANG $^{1}$, Hai-feng SONG ${ }^{1, *}$ \\ ${ }^{1}$ Department of Pharmacology and Toxicology, Beijing Institute of Radiation Medicine, Beijing 100850, China; ${ }^{2}$ Drug Clinical Trial Insti- \\ tution, Beijing Anzhen Hospital of Capital Medical University, Beijing 100029, China; ${ }^{3}$ Department of Cardiovascular Surgery, Xijing \\ Hospital, Fourth Military Medical University, Xi'an 710032, China
}

\begin{abstract}
Aim: DNAs containing unmethylated CpG motifs can stimulate innate and adaptive immunity. The aim of this study was to investigate the immunostimulatory and anti-neoplasm effects of a novel $\mathrm{CpG}$ oligodeoxynucleotide, ODN10, in tumor-bearing mice.

Methods: B16 melanoma-bearing C57BL/6 mice were administered ip or sc with ODN10 or conventional CpG ODN1826 on the indicated days post inoculation. The animal survival rate and the inhibitory effect on tumor growth were observed in vivo. B and T lymphocyte proliferation, natural killing cell cytotoxicity and the phagocytic ability of peritoneal macrophages from the animals were determined using $\left[{ }^{3} \mathrm{H}\right]$-thymidine incorporation assay, $4-\mathrm{h}^{51} \mathrm{Cr}$ release assay and neutral red chromometry method, respectively. The serum levels of IL-12, IL-4, and IgE were quantified using ELISA assays. Histological examination of tumor tissues was performed after HE staining, and the expression of PCNA, CD63, and CD80 in tumor tissues was analyzed with immunohistochemistry.

Results: ODN10 (1, 5, and $25 \mathrm{mg} / \mathrm{kg})$ significantly inhibited the growth and metastasis of the tumor, and significantly prolonged the survival of tumor-bearing mice, as compared with ODN1826. The immune status was suppressed in tumor-bearing mice. Both ODN10 and ODN1826 significantly reversed the suppressed immunoactivities in tumor-bearing mice, which included promoting B and T lymphocyte proliferation, enhancing NK cell and peritoneal macrophage activities, inducing IL-12 secretion and inhibiting IL-4 and IgE secretion. Further, CpG ODNs decreased PCNA and CD63 expression while induced expression of CD80. ODN10 presented more potent activity, and displayed the most prominent immunostimulatory potential.

Conclusion: ODN10 produces prominent immunomodulatory effects on cellular immunity in tumor-bearing mice, which might help reverse the established Th2-type responses to the Th1-type responses, thus may be used as a potent anti-tumor immunotherapy agent or adjuvant.
\end{abstract}

Keywords: CpG oligodeoxynucleotide; ODN10; ODN1826; melanoma; cancer; immunotherapy; cellular immunity

Acta Pharmacologica Sinica (2012) 33: 1047-1054; doi: 10.1038/aps.2012.54; published online 25 Jun 2012

\section{Introduction}

The immune system of vertebrates is comprised of two complementary systems, the innate and the adaptive. In the innate system, dendritic cells (DCs) are the major antigen presenting cells to $T_{h 0} T$ cells in lymph nodes that polarise into $T_{h 1}$ and $T_{h 2}$ cells, which subsequently produce different cytokines. Polarised $\mathrm{T}_{\mathrm{h} 1}$ cells produce interleukin (IL)-2, IL-12, and interferon (IFN)- $\gamma$, while polarised $T_{\mathrm{h} 2}$ cells produce IL-4, IL-5, IL-6, IL-10, and IL-13. In healthy individuals, there is a $T_{h 1} / T_{h 2}$ cytokine balance, whereas in the tumor-bearing individual, $\mathrm{T}_{\mathrm{h} 2}$ cytokine

\footnotetext{
\# The first three authors contributed equally to this work.

* To whom correspondence should be addressed.

E-mail songhf@vip.163.com

Received 2011-11-03 Accepted 2012-04-23
}

synthesis is increased. As such, researchers have tried to upregulate the $T_{h 1}$ population and maintain the balance of $T_{h 1} / T_{h 2}$ to reverse the abnormal immune status in tumor patients ${ }^{[1]}$.

Bacterial and synthetic DNAs containing unmethylated CpG motifs were known to stimulate innate and adaptive immunity due to their interesting immunostimulatory properties in a number of vertebrates. The recognition of CpG DNAs by immune cells was mediated by Toll-like receptor 9 (TLR9), a receptor mainly expressed by B cells and plasmacytoid DC cells in humans and macrophages in mice ${ }^{[2]}$. TLR9-stimulated immune cells showed increased expression of co-stimulatory molecules, resistance to apoptosis, and up-regulation of $\mathrm{T}_{\mathrm{h} 1^{-}}$ promoting chemokines and cytokines such as IL-2, IL-12, and IFN- $\gamma^{[3]}$. In vivo, CpG DNAs were strong $\mathrm{T}_{\mathrm{h} 1}$-inducing adjuvants $^{[4-8]}$. 
Recently, CpG oligodeoxynucleotide (ODN)s have been explored for use as an anti-cancer therapy due to their immunostimulatory properties, such as inducing the release of large amounts of type I IFN, inhibition of the synthesis of $T_{h 2}$ cytokines, induction of $\mathrm{T}_{\mathrm{h} 1}$ cytokine synthesis, and the activation of NK cell cytotoxicity or cytotoxic T lymphocyte (CTL) precursors into anti-tumor CTL cells ${ }^{[9-13]}$. Further, CpG ODNs enhanced the anti-tumor efficacy of monoclonal antibodies or cancer vaccines when used as an adjuvant in animal tumor models ${ }^{[11,14]}$. CpG ODNs alone were also capable of triggering potent anti-tumor immune responses against various experimental tumors, for example, lymphoma, melanoma, colon tumor, glioma, and neuroblastoma ${ }^{[15-21]}$.

We previously investigated the relationship between primary and secondary structures of CpG ODNs and their immunostimulatory effects on murine spleen cells and found that specific structures of CpG ODNs could vary greatly in their ability to induce host immunity in mice ${ }^{[22]}$. We screened several novel CpG ODN sequences to identify those with superior immunostimulatory activities relative to the conventional linear control CpG ODN1826, which is a strong immune activator that induces protective and curative $\mathrm{T}_{\mathrm{h} 1}$ responses against infections and tumors in vivo in mice $\mathrm{e}^{[9,20,21,23-25]}$. Among them, ODN10, which possessed a holistic, self-complementary palindrome structure and promised the strongest immunostimulatory potential, was chosen for use in the present study. The immunostimulatory potential of ODN10 was evaluated by its activation of NK cells, T cells, B cells, macrophages, and the helper $\mathrm{T}$ cell related cytokine secretion profile in malignant melanoma-bearing mice. To characterise the most important outcome of immunomodulatory therapy, the in vivo anti-tumor efficacy of ODN10 was investigated as well. The results suggested that ODN10, a novel CpG ODN with self-palindrome structure, displayed outstanding anti-tumor activity superior to that of other conventional CpG-containing immunostimulatory ODN sequences. In addition, the anti-tumor activity of ODN10 may be attributed to its powerful immunoregulatory potential to reverse established $T_{h 2}$-type responses to a $T_{h 1}$ type response.

\section{Materials and methods CpG ODNs}

Purified, single-stranded, phosphorothioated ODNs containing CpG motifs were synthesised by Sangon Biotech Company (Shanghai, China). The CpG ODNs used in this study were as follows: ODN10, 5'-TCCATGACGTTTTAAAACGTCATGGA-3', ODN1826, 5'-TCCATGACGTTCCTGACGTT-3', nonCpG ODN control, 5'-TGCTGCTTTTGTGCTTTTGTGCTT-3'. Schematic diagrams of the secondary structure of ODN1826 and ODN10 are shown in Figure 1. All ODNs were diluted in phosphate-buffered saline (PBS) $(0.1 \mathrm{~mol} / \mathrm{mL}, \mathrm{pH} 7.3)$ and stored at $4^{\circ} \mathrm{C}$ before use.

\section{Cells and cell lines}

Spleen cells from 6- to 8-week-old C57BL/6 mice were cultured in RPMI-1640 medium supplemented with 10\% (v/v)

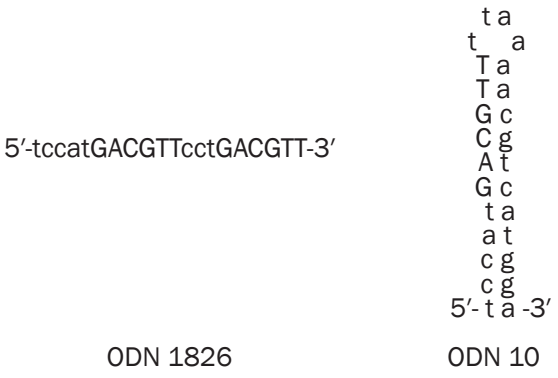

Figure 1. Schematic diagram of ODN1826 and ODN10. ODN1826 (left) is a typical linear type ODN. ODN10 (right) possesses a holistic, selfcomplementary palindrome structure.

fetal bovine serum (FBS; GIBCO, Detroit, MI, USA) and antibiotics (100 IU/mL of penicillin and $100 \mathrm{IU} / \mathrm{mL}$ of streptomycin). The B16.F1 melanoma cells, which are syngeneic to $\mathrm{C} 57 \mathrm{BL} / 6$ mice, were maintained in vitro as previously described $^{[26,27]}$. Yeast artificial chromosome-1 (YAC-1) cells were kindly provided by Prof Wen-xia ZHOU (Institute of Pharmacology and Toxicology, Beijing, China).

\section{Experimental animals}

Female C57BL/ 6 mice (6-8 weeks of age, 20.0 $2.0 \mathrm{~g}$ ), were purchased from the Experimental Animal Raising Center of the Academy of Military Medical Sciences (Beijing, China). All animal experiments and protocols were performed strictly in accordance with the Guide for the Care and Use of Laboratory Animals of the National Institutes of Health.

\section{Tumor models}

Exponentially growing B16.F1 melanoma cells were harvested when their viability exceeded $95 \%$, which was determined by trypan blue staining. The cells were resuspended in 0.2 $\mathrm{mL}$ PBS and were injected subcutaneously (sc) at the dosage of $2 \times 10^{6}$ cells/animal into the right axilla of C57BL/ 6 mouse. The cells resuspended in $0.2 \mathrm{~mL}$ PBS were injected intraperitoneally (ip) into another group of individuals at a dose of $2 \times 10^{5}$ cells/animal.

\section{Dose determination}

Tumor-bearing mice (sc inoculated) were randomly divided into various treatment groups (10 animals in each group) and then treated ip with PBS or ODN10 on 9, 12, and $15 \mathrm{~d}$ post inoculation at the indicated doses $(1,5$, or $25 \mathrm{mg} / \mathrm{kg})$. Mice were checked daily for tumor growth. Tumor volume was calculated using the formula $1 / 2 a \times b^{2}$, where $a$ and $b$ are the long and short diameters (in $\mathrm{mm}$ ), respectively.

\section{Survival assay}

Tumor-bearing mice (ip inoculated) were divided randomly into various treatment groups (10 animals in each group). On 0,3 , and $7 \mathrm{~d}$ post inoculation and weekly thereafter, the mice received ip treatment of ODN10, ODN1826, or non-CpG ODN and PBS control, with all ODNs administered at the same dos- 
age of $5 \mathrm{mg} / \mathrm{kg}$. The survival of mice was checked daily until all animals died.

\section{In vivo inhibitory effect on tumor growth}

Subcutaneously inoculated tumor-bearing mice were divided randomly into four treatment groups (10 animals in each group) and then treated ip with various ODNs (ODN10, ODN1826, or non-CpG ODN, all at the dose of $5 \mathrm{mg} / \mathrm{kg}$ ) or PBS on $\mathrm{d} 9$ (when the tumor size reached approximately $5 \mathrm{~mm}$ in diameter), 12, 16, and 19 post tumor inoculation. Mice were checked daily for tumor growth, and the mice were sacrificed on $\mathrm{d} 20$ post inoculation, when the tumor size from mice in the PBS control group reached approximately $25 \mathrm{~mm}$ on the long diameter. The tumors and spleens were collected from individual animals and weighed. The oxter lymph nodes and the ascites were collected for further study. The serum was collected into $2 \mathrm{~mL}$ microtubes and stored at $-70^{\circ} \mathrm{C}$ until measurement. The inhibitory effect of the ODNs on tumor growth in vivo was evaluated by the tumor growth inhibitory rate $(\mathrm{TGI} \%)$, which was calculated by the formula $\left(W_{0}-W\right) /$ $W_{0} \times 100 \%$, where $W$ represented the tumor weight of the ODNtreated groups, and $W_{0}$ was that of the PBS control group.

\section{B and T lymphocyte proliferation assay}

Proliferation of mouse B cells and T cells from tumor-bearing mice treated with different ODNs or the control, as well nontumor-bearing mice was determined by a $\left[{ }^{3} \mathrm{H}\right]$-thymidine incorporation assay as described ${ }^{[28]}$. Briefly, splenocytes $\left(2 \times 10^{5}\right.$ cells $)$ harvested from tumor-bearing mice or the normal mice were loaded in a 96-well culture plate with or without 5 $\mu \mathrm{g} / \mathrm{mL}$ ConA or LPS (Sigma, St Louis, MO, US) and cultured at $37^{\circ} \mathrm{C}$ in a $5 \% \mathrm{CO}_{2}$ atmosphere in RPMI- 1640 for $72 \mathrm{~h}$, with 1 $\mu \mathrm{Ci}$ of $\left[{ }^{3} \mathrm{H}\right]$-thymidine $\left({ }^{3} \mathrm{H}\right]$-TdR, Institute of Atomic Energy, Beijing, China) added for the last $18 \mathrm{~h}$. The cells were harvested, and the radioactivity was measured using a microBeta liquid scintillation counter (PerkinElmer, Waltham, MA, USA).

\section{NK-mediated cytotoxicity assay}

The NK cytotoxicity of splenocytes was detected with a $4 \mathrm{~h}$ ${ }^{51} \mathrm{Cr}$ release assay as previously described ${ }^{[27]}$. Briefly, splenocytes were harvested from tumor-bearing mice or nontumor-bearing mice to serve as effector cells. One million YAC-1 cells/well (serving as target cells) were labelled with $50 \mu \mathrm{Ci}$ of ${ }^{51} \mathrm{Cr}$ for $1 \mathrm{~h}$ at $37^{\circ} \mathrm{C}$ in a 96-well microplate and were then washed 3 times and incubated for $4 \mathrm{~h}$ with effector cells (with an E:T ratio of 50:1). Thereafter, supernatants were harvested, and the radioactivity was measured using a microBeta liquid scintillation counter (PerkinElmer, Waltham, MA, USA). The results are expressed as the mean percentage of specific lysis (triplicate wells) $\pm \mathrm{SD}$, where percent specific lysis $(\%)=($ experimental countstarget cell spontaneous release counts)/(maximal release counts-target cell spontaneous release counts) $\times 100 \%$. Spontaneous lysis was measured in wells containing only target cells, whereas maximum lysis was measured from the wells containing target cells incubated with $10 \%$ SDS.

\section{Macrophage phagocytosis assay}

The phagocytic ability of peritoneal macrophages was examined with a neutral red chromometry method strictly as described $^{[29]}$. The data shown are representative of two replicate experiments.

\section{Cytokines and chemokines ELISAs}

Serum IL-12, IL-4, and IgE levels were measured twice by ELISA according to the manufacturer's instructions (IL-12 P40 and IL-4 kits were purchased from R\&D Systems (Minneapolis, MN, USA), and the IgE kit was purchased from BETHYL (Montgomery, TX, USA). The plate reader was calibrated to the manufacturer's specifications, and the absorbance $(450 \mathrm{~nm})$ of the contents of each well was determined.

\section{Histopathology and immunohistochemistry}

After dissection, specimens of tumor, spleen, and oxter lymph nodes were fixed for 1 week in $4 \%$ buffered paraformaldehyde, embedded in paraffin, and stained with hematoxylin and eosin (H\&E staining). PCNA, CD63, and CD80 expression levels in the tumor tissues were analysed by immunohistochemistry. Briefly, the paraffin-embedded sections were dewaxed and rehydrated. Antigen retrieval was performed in citrate buffer ( $\mathrm{pH}$ 6.0) using the microwave technique. Endogenous peroxidase activity was quenched by $30 \%$ hydrogen peroxide in distilled water for 5-10 $\mathrm{min}$ and then washed in PBS. Primary antibodies (mouse anti-mouse monoclonal antibodies against PCNA, rabbit anti-mouse monoclonal antibodies against CD63 and CD80), the Mouse/Rabbit IgG SABC kit, and 3,3'-diaminobenzidine (DAB) solution were purchased from Wuhan Boster Biotechnology (Wuhan, China). Sections were sequentially incubated with primary antibody, the biotinylated anti-mouse/rabbit antibody, and the streptavidin peroxidase reagent. Peroxidase activity was detected with DAB solution. The same process performed without the primary antibody was used as a control. Sections were weakly counterstained with hematoxylin.

\section{Statistical analysis}

The data are shown as the mean $\pm \mathrm{SD}$, and statistical significance was determined with the SPSS (v 10.0) software with Student's $t$ test or analysis of variance (one-way ANOVA). Survival data were analysed by the Mantel-Cox logrank test. $P<0.05$ was considered to be statistically significant.

\section{Results}

\section{Inhibitory effect on tumor growth in vivo}

Inoculated B16 melanoma cells $\left(2 \times 10^{6}\right.$ cells/mouse) led to invasive tumors in $100 \%$ of the C57BL/ 6 mice. At $\mathrm{d} 8$, when all mice presented a single mass (tumor size of ca $4 \mathrm{~mm}$ in diameter) in the right oxter, $\mathrm{CpG}$ ODNs were administered ip at the indicated doses $(1,5$, and $25 \mathrm{mg} / \mathrm{kg})$ on $\mathrm{d} 9,12$, and 14 after tumor inoculation. Mice were checked daily for tumor growth. The results showed that ODN10 inhibited the growth of the tumor in vivo in a dose-dependent manner (Figure 2A). Compared with the PBS control group, TGI rates of various 

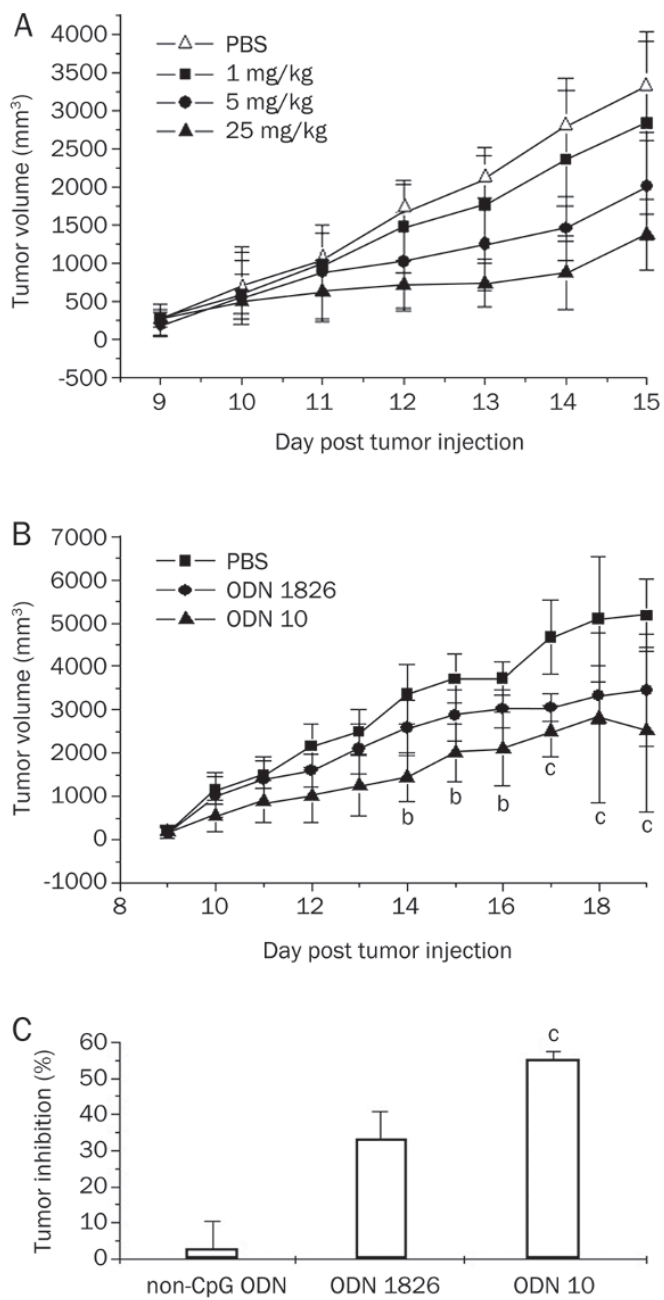

Figure 2. Effect of $\mathrm{CpG}$ ODNs on the growth of B16 melanoma in mice. C57BL/ 6 mice were inoculated SC with $2 \times 10^{6} \mathrm{~B} 16$ melanoma cells on d 0 . Mice were treated with ODN1826 (positive control) and ODN10 at the indicated times and doses. (A) Dose-dependent effect of ODN10 (on d 9, 12,14 ; at doses of 1,5 , and $25 \mathrm{mg} / \mathrm{kg}$ ). (B) Tumor growth curve on d 20 post tumor inoculation at doses of $5 \mathrm{mg} / \mathrm{kg}$ at the indicated times (on d 9 , 12, 16, and 19). ${ }^{b} P<0.05,{ }^{c} P<0.01$ vs PBS-treated mice; (C) Tumor growth inhibitory rate (TGI\%) on $d 20 .{ }^{c} P<0.01$ vs ODN1826-treated mice. There were 10 mice in each group. The data shown represent two replicate experiments.

dosage groups $(1,5$, and $25 \mathrm{mg} / \mathrm{kg})$ were $13.4 \%(P<0.05)$, $35.5 \%(P<0.01)$, and $48.3 \%(P<0.01)$ on $\mathrm{d} 15$, respectively. Figure $2 \mathrm{~B}$ showed that the $\mathrm{CpG}$ ODN, at the same dosage level of $5 \mathrm{mg} / \mathrm{kg}$, could inhibit the tumor proliferation significantly $(P<0.05)$ from $\mathrm{d} 14$, and more significantly $(P<0.01)$ from $\mathrm{d}$ 17. On $d 20$, the TGI rate of the ODN10 treatment group was $51.1 \%$, which is remarkably superior to that of ODN1826 $(33.2 \%, P<0.01)$. The non-CpG ODN hardly showed any anti-tumor activity (Figure 2C). Compared with the positive ODN1826, ODN10 showed higher antitumor activity. ip on $\mathrm{d}$. The $\mathrm{CpG}$ or non-CpG ODN was administered ip on d 0, 3, 7, and weekly thereafter. As shown in Figure 3, on d 22, the survival rates of non-CpG ODN, ODN1826, and ODN10 groups were $0 \%, 20 \%$, and $70 \%$, respectively, compared with the PBS control group. CpG ODN can significantly prolong the survival of mice with B16 melanoma. ODN10 showed more effectiveness than ODN1826 and its potent antitumor activity in vivo was illustrated again.

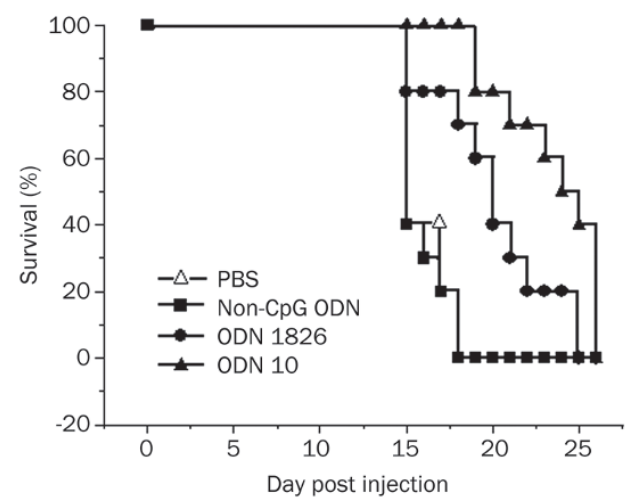

Figure 3. ODN10 prolonged the survival of mice implanted with B16 melanoma ( $2 \times 10^{5}$ cells/mouse). On d 0, 3, 7, and weekly thereafter, the mice received $5 \mathrm{mg} / \mathrm{kg}$ ip of the indicated ODN and were observed for survival. There were 10 mice in each group. The data shown represent two replicate experiments.

\section{In vivo immunostimulatory activities of ODN10}

We investigated the difference between the immune states of the tumor-bearing mice and normal mice, with the measurement indicators included in the study being the proliferation potential of B and T lymphocytes, NK cytotoxicity of splenocytes, and the phagocytic ability of peritoneal macrophages. The results showed that all of the above-mentioned immune activities were suppressed in tumor-bearing mice compared to their normal counterparts $(P<0.05)$. However, the $\mathrm{CpG}$ immunostimulatory sequences significantly reversed the immunosuppression status in tumor-bearing mice. Among them, the ODN10 displayed the most prominent immunostimulatory potential (Figure 4).

As to the influences on chemokine or cytokine secretion, CpG ODN treatment is known to reverse established $\mathrm{T}_{\mathrm{h} 2}$ responses to $\mathrm{T}_{\mathrm{h} 1}$-type responses ${ }^{[30,31]}$. In the present study, IL-12 and the IL-4 were selected as the indicator cytokines, where the former is a $\mathrm{T}_{\mathrm{h} 1}$-type cytokine, while the latter is a $\mathrm{T}_{\mathrm{h} 2}$-type cytokine. As a result of induction of IL-4, B cells switch to IgE synthesis and inhibit IgG antibody synthesis; therefore, IgE levels in mice were also monitored as a marker of $\mathrm{T}_{\mathrm{h} 1}-\mathrm{T}_{\mathrm{h} 2}$ response type conversion. The results showed that in the absence of CpG ODN treatment, serum from tumorbearing mice contained markedly higher IL-4 and IgE levels and lower IL-12 levels than normal mice, suggesting a predominantly $\mathrm{T}_{\mathrm{h} 2}$ polarisation, while the CpG ODN treatment 

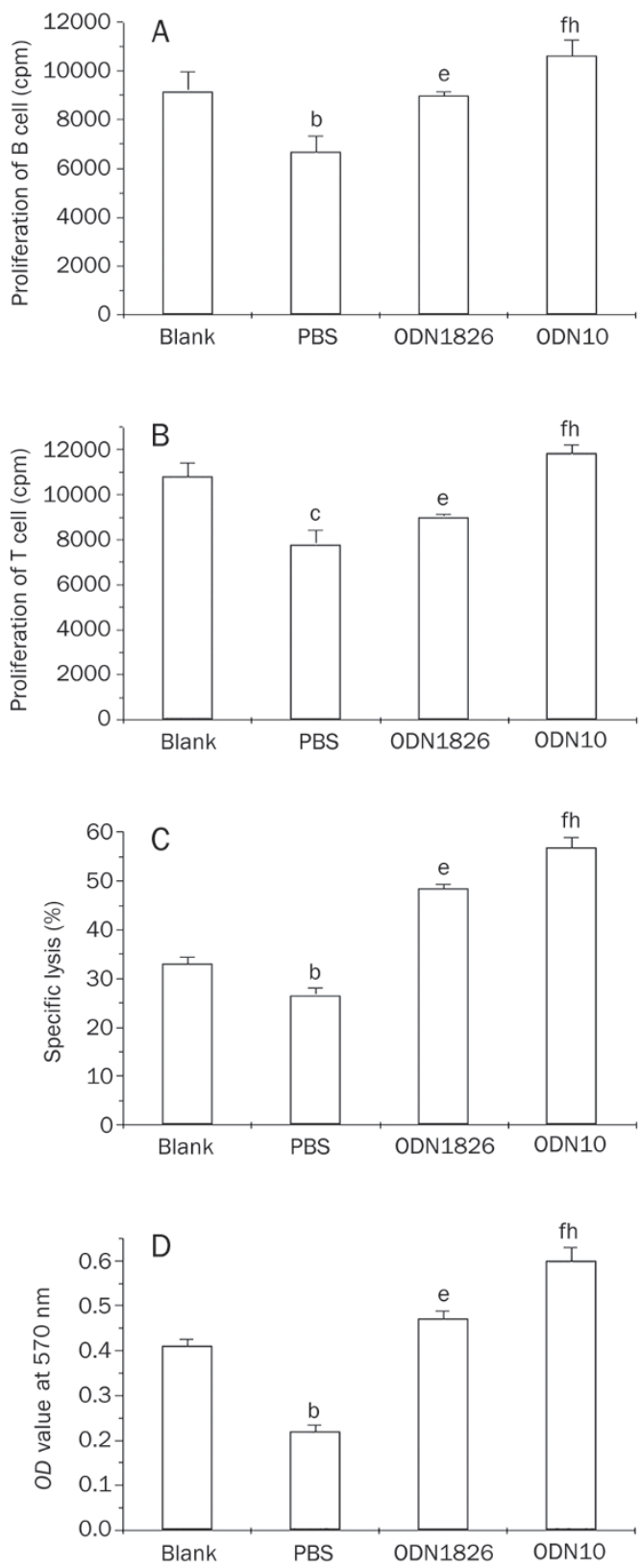

Figure 4. Immunostimulatory activity of $\mathrm{CpG}$ ODNs in tumor-bearing mice. Splenocytes were from the ODN-treated tumor-bearing mice (ODN1826 or ODN10), age-matched PBS-treated tumor-bearing mice (PBS), or agematched normal mice (Blank). The peritoneal macrophages were also from the above groups. (A) Proliferation of the LPS-activated B cells. (B) Proliferation of the ConA-activated T cells. (C) NK cytotoxicity of splenocytes. (D) Phagocytic ability of peritoneal macrophage. ${ }^{b} P<0.05,{ }^{\mathrm{C}} P<0.01$, PBS-treated tumor-bearing mice vs normal mice; ${ }^{e} P<0.05,{ }^{f} P<0.01$, ODNtreated tumor-bearing mice vs PBS-treated tumor-bearing mice; ${ }^{h} P<0.05$, ODN10-treated tumor-bearing mice vs ODN1826-treated tumor-bearing mice. There were 10 mice in each group. The data shown represent two replicate experiments.

markedly reversed the established $T_{h 2}$ responses to $T_{h 1}$-type responses in tumor-bearing mice, characterised by the significant up-regulation of IL-12 and down-regulation of IL-4 and
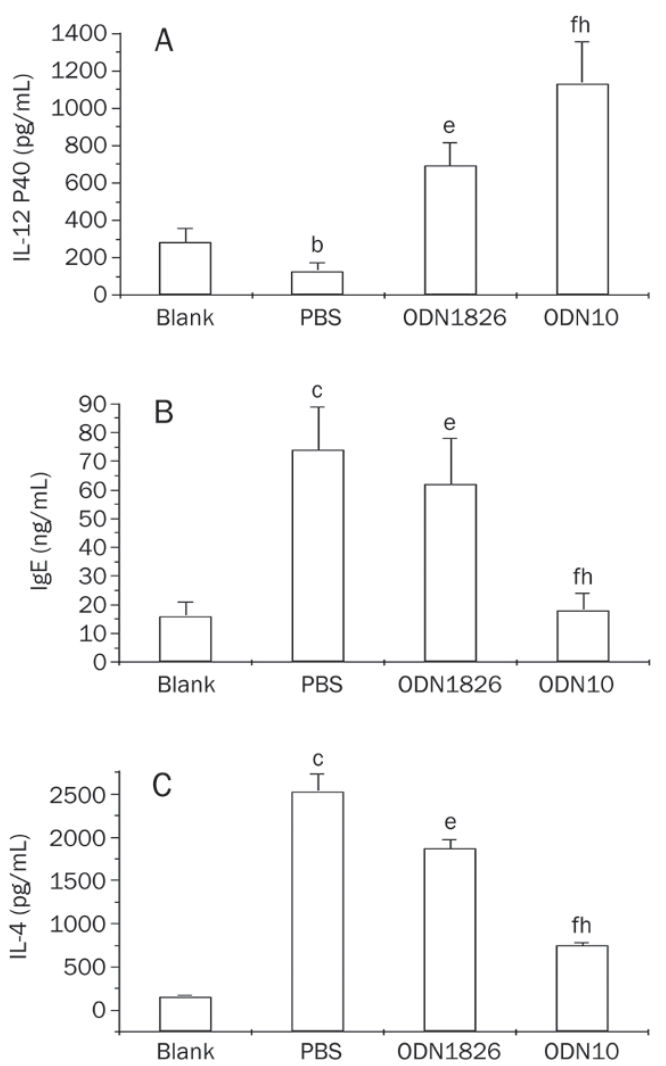

Figure 5. Induction of IL-12 P40 (A), IgE (B), or IL-4 (C) production by CpG ODN in mice. Sera were from the ODN-treated tumor-bearing mice (ODN1826 or ODN10), age-matched PBS-treated tumor-bearing mice (PBS), or age-matched normal mice (Blank). ${ }^{b} P<0.05,{ }^{c} P<0.01$, PBStreated tumor-bearing mice vs normal mice; ${ }^{e} P<0.05,{ }^{f} P<0.01$, ODNtreated tumor-bearing mice vs PBS-treated tumor-bearing mice; ${ }^{h} P<0.05$, ODN10-treated tumor-bearing mice vs ODN1826-treated tumor-bearing mice. There were 10 mice in each group. The data shown represent two replicate experiments.

IgE. Again, ODN10 had more significantly robust immunostimulatory effects compared with ODN1826 (Figure 5).

\section{Pathobiology and immunohistochemistry analysis}

Figure 6 shows the H\&E staining of tissue sections from formalin-fixed tumors obtained $20 \mathrm{~d}$ after tumor injection. PBS-treated tumors exhibited a solid growth pattern without necrosis. ODN10 treatment could make the tumor cell exhibit extensive cellular polymorphisms, including cavitate nuclei, swollen nuclei, and apoptosis. Figure 7 shows the H\&E staining of tissue sections from spleen or oxter lymph nodes. The PBS group presented with large-scale necrosis and vasal infarct by metastatic melanoma masses in the spleen and metastatic melanoma in the vessels of oxter lymph nodes. Both the spleen and oxter lymph nodes were normal in the $\mathrm{CpG}$ ODN10 treatment group.

PCNA was expressed exclusively in tumor tissues of the melanoma mice, and ODN10 treatment decreased PCNA expression (Figure 8A-C). Expression of CD63 (Figure 8D-F), a melanoma metastasis-associated antigen, was similar to PCNA. On the contrary, ODN10 could induce expression of 

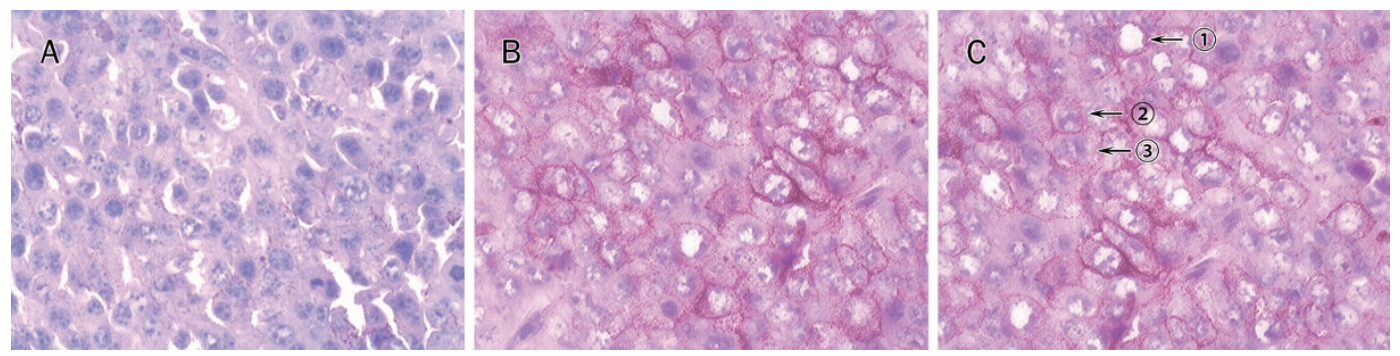

Figure 6. Tumor tissues were fixed in $4 \%$ buffered paraformaldehyde, embedded in paraffin and analysed histologically by H\&E staining ( $\times 132)$. PBStreated tumors showed a solid growth pattern without necrosis (A). Tumors treated with ODN1826 (B) or ODN10 (C) exhibited extensive cellular polymorphism, including cavitate nuclei (arrow(1)), swollen nuclei (arrow(2)), and apoptosis (arrow(3)).
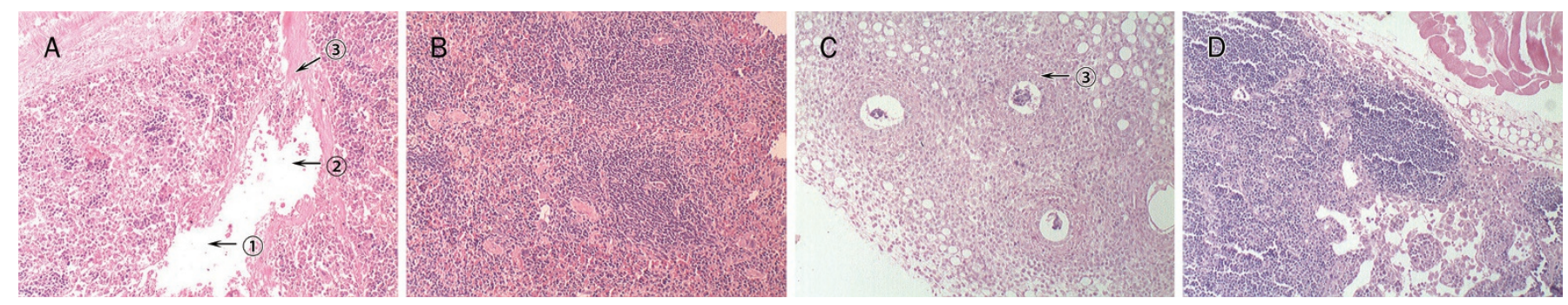

Figure 7. Histological analysis of spleen and oxter lymph nodes (HE staining, $\times 40$ ). The PBS-treated group presented with large-scale necrosis (arrow(1) and vasal infarct (arrow(2) by a metastatic melanoma mass (arrow(3)) in the spleen (A), whereas the ODN10 treatment group was normal (B). The PBStreated group showed a metastatic melanoma mass in the vessel of oxter lymph nodes (arrow(3) (C), whereas the ODN10 treatment group was normal (D).

CD80, a cell-surface co-stimulatory molecule, in tumor tissues of melanoma-bearing mice (Figure 8G-I). The results from pathobiology and immunohistochemistry analysis indicated that CpG ODNs could activate the host anti-tumor immune response to inhibit the growth and metastasis of melanoma tumors in mice, and the novel ODN10 presented more potent activity.

\section{Discussion}

Bacterial and synthetic CpG DNAs promote B cell proliferation, immunoglobulin production and the secretion of a number of cytokines, including IL-6, IL-12, IFN- $a$, and IFN- $\gamma$, from B cells, monocytes/macrophages, DC cells and NK cells. ODN1826 is known as a strong immune activator that induces protective and curative $T_{h 1}$ responses against infections and tumorigenesis in vivo for mice ${ }^{[10,23-25]}$.

Our previous studies showed that ODN10, a novel CpG ODN containing a holistic self-complementary palindrome structure (5'-TCCATGACGTTTTAAAACGTCATGGA-3'), possesses more potent immunostimulatory effects than a conventional CpG ODN (ODN1826), characterised by inducing a high level of IL-12 and IFN-a secretion and potentiating NK cell lytic activity ${ }^{[22]}$. We presume that their immunostimulatory properties may play roles in the CpG ODN-induced inhibition of B16 melanoma growth.

In our study, we found that the proliferation of B cells and $\mathrm{T}$ cells, NK cytotoxicity of splenocytes, and the phagocytic ability of peritoneal macrophages in tumor-bearing mice were lower than those in normal mice. Additionally, we also found that serum from tumor-bearing mice contained markedly higher IL-4 and IgE levels and lower IL-12 levels than normal mice, suggesting a predominantly $\mathrm{T}_{\mathrm{h} 2}$ polarisation. These phenomena indicated that the burden of the tumor might impair the function of an individual's innate and adaptive immune system. In tumor-bearing individuals, when IL-4, a type of $\mathrm{T}_{\mathrm{h} 2}$ cytokine, is produced at a higher level than normal, it can induce the IL-4 response genes in T cells and also shut off IgG synthesis by B cells and induce IgE synthesis. Under these conditions, the high $\mathrm{T}_{\mathrm{h} 2}$ cytokine level is dominant in the individual, an allergy-like inflammatory condition develops, the adaptive immune response is inhibited, and tumor development and progression continue. The increased IgE levels in cancer patients reflect impaired cellular immunity ${ }^{[1]}$.

Our results also showed that the activities of the above-mentioned immune cells increased remarkably from the CpG ODN treatment, especially from ODN10 treatment. Meanwhile, we found that the antitumor activity of CpG ODNs was increased along with their immunomodulatory activity, for example, prolonged survival of mice with B16 melanoma, and inhibition of the tumor growth. Pathobiology and immunohistochemistry analysis also indicated that ODN10 could activate the host anti-tumor immune response to inhibit the in vivo growth and metastasis of melanoma tumors in mice.

Our study showed that ODN10 had potent antitumor activity, which was positively correlated with its superior immunomodulatory effects, including promoting B and T lymphocyte proliferation, enhancing NK cell and peritoneal macrophage activity, inducing IL-12 secretion, and inhibiting IL-4 and 


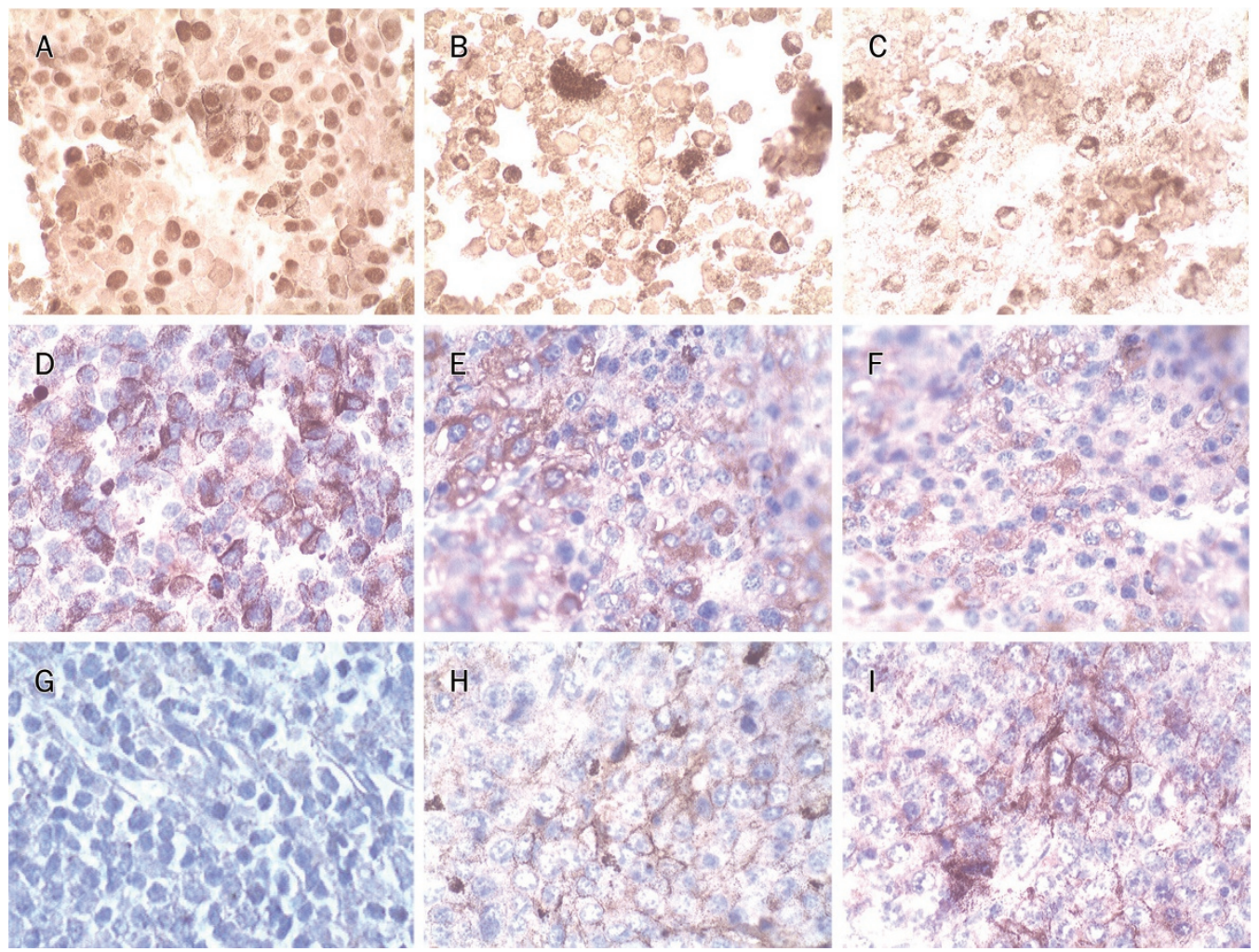

Figure 8. Immunohistochemistry analysis of tumor tissues $(\times 132)$. Paraffin-embedded sections were dewaxed and rehydrated. Endogenous peroxidase activity was quenched by $30 \%$ hydrogen peroxide. Antigen retrieval was achieved by microwave treatment. Sections were sequentially incubated with primary antibody, the biotinylated anti-mouse/rabbit antibody, and the streptavidin peroxidase reagent. Peroxidase activity was detected with DAB solution, and sections were weakly counterstained with hematoxylin. PCNA was strongly expressed in PBS-treated mice (A), whereas it was remarkably decreased in ODN1826 (B) or ODN10-treated mice (C). CD63 was strongly expressed in PBS-treated mice (D), whereas it was remarkably decreased in ODN1826 (E) or ODN10-treated mice (F). The tumor stained negatively for co-stimulatory molecules CD80 in PBS-treated mice (G), whereas CD80 was expressed in ODN1826 (H) or ODN10-treated mice (I).

IgE secretions. We presume that ODN10 can induce pDCmediated cellular adaptive immunity to release large amounts of type I IFN; the latter stimulates $\mathrm{T}_{\mathrm{h} 1}$ cells to secret IL-2 and IL-12, which induce the CTL precursors to differentiate into antitumor CTL cells and also induce B cells to synthesise antitumor IgG antibodies. Meanwhile, ODN10 can stimulate NK cells responsible for spontaneous cytotoxicity of a variety of tumor cells and can activate macrophages, scavengers of dead cell debris. In addition, our study also demonstrated that the novel ODN10 can reverse established $T_{h 2}$ responses to $T_{h 1}$-type responses and change an individual's tumor microenvironment, which contributes to identifying and killing tumor cells.

In summary, our experimental results demonstrated that the novel ODN10 has more potent immunomodulatory and antitumor activity towards melanoma than other ODNs, and as such, displays a promising application in cancer immunotherapy in mice. Our study suggests that the palindrome may be essential to the immunostimulatory potency of a CpG ODN and contributes to its antitumor activity.

\section{Acknowledgements}

This work was supported by the National Natural Science Foundation of China (grant number 30572197) and the
National High Biotechnology Development Program of China (grant number 2003AA2Z347B).

We gratefully acknowledge Prof Wen TAN (Cancer Institute \& Hospital, Chinese Academy of Medical Sciences) for her excellent technical assistance.

\section{Author contribution}

Hai-yan DU, Li-hou DONG, Bi-jun ZHAO, Zhong-ming TANG, and Hai-feng SONG designed the research study; Jie FU, Lun OU, Xiao SUN, and Na LI performed the research; Qing-qing WANG and Fang CHEN analysed the data; Haiyan DU, Li-hou DONG, and Bi-jun ZHAO wrote the manuscript.

\section{References}

1 Yechiel B. Molecular immunological approaches to biotherapy of human cancers - A review, hypothesis and implications. Anticancer Res 2006; 26: 1113-34.

2 Hemmi H, Takeuchi O, Kawai T, Kaisho T, Sato S, Sanjo H, et al. A Tolllike receptor recognizes bacterial DNA. Nature 2000; 408: 740-5.

3 Krieg AM. CpG motifs in bacterial DNA and their immune effects. Annu Rev Immunol 2002; 20: 709-60.

4 Klinman DM. Immunotherapeutic uses of CpG oligodeoxynucleotides. 
Nat Rev Immunol 2004; 4: 249-58.

5 Latz E, Schoenemeyer A, Visintin A, Fitzgerald KA, Monks BG, Knetter $\mathrm{CF}$, et al. TLR9 signals after translocating from the ER to CpG DNA in the lysosome. Nat Immunol 2004; 5: 190-8.

6 Verthelyi D, Klinman DM. Immunoregulatory activity of CpG oligonucleotides in humans and nonhuman primates. Clin Immunol 2003; 109: 64-71.

7 Marshall JD, Fearon K, Abbate C, Subramanian S, Yee P, Gregorio J, et al. Identification of a novel CpG DNA class and motif that optimally stimulate B cell and plasmacytoid dendritic cell functions. J Leukocyte Biol 2003; 73: 781-92.

8 Vollmer J, Weeratna R, Payette P, Jurk M, Schetter C, Laucht M, et al. Characterization of three $\mathrm{CpG}$ oligo-deoxynucleotide classes with distinct immunostimulatory activities. Eur J Immunol 2004; 34: 25162.

9 Brunner C, Seiderer J, Schlamp A, Bidlingmaier M, Eigler A, Haimerl $\mathrm{W}$, et al. Enhanced dendritic cell maturation of TNF-alpha or cytidinephosphate-guanosine DNA drives T cell activation in vivo and therapeutic anti-tumor immune responses in vitro. J Immunol 2000; 165: 6278-86.

10 Ballas ZK, Krieg AM, Warren T, Rasmussen W, Davis HL, Waldschmidt $M$, et al. Divergent therapeutic and immunologic effects of oligodeoxynucleotides with distinct CpG ODN motifs. J Immunol 2001; 167: 4878-86.

11 Wooldridge JE, Ballas ZK, Krieg AM, Weiner GJ. Immunostimulatory oligodeoxynucleotides containing CpG motifs enhance the efficacy of monoclonal antibody therapy of lymphoma. Blood 1997; 89: 2994-8.

12 van Ojik HH, Bevaart L, Dahle CE, Bakker A, Jansen $\mathrm{MJH}$, van Vugt $M J$, et al. CpG-A and B oligodeoxynucleotides enhance the efficacy of antibody therapy by activating different effector cell populations. Cancer Res 2003; 63: 5595-600.

13 Heckelsmiller K, Beck S, Rall K, Sipos B, Schlamp A, Tuma E, et al. Combined dendritic cell and $\mathrm{CpG}$ oligonucleotide-based immune therapy cures large murine tumors that resist chemotherapy. Eur J Immunol 2002; 32: 3235-45.

14 Davila E, Celis E. Repeated administration of cytosine-phosphorothiolated guanine-containing oligonucleotides together with peptide/ protein immunization results in enhanced CTL responses with antitumor activity. J Immunol 2000; 165: 539-47.

15 Blazar BR, Krieg AM, Taylor PA. Synthetic unmethylated cytosinephosphateguanosine oligodeoxynucleotides are potent stimulators of antileukemia responses in naive and bone marrow transplant recipients. Blood 2001; 98: 1217-25.

16 Sharma S, Karakousis CP, Takita H, Shin K, Brooks SP. Intra-tumoral injection of $\mathrm{CpG}$ results in the inhibition of tumor growth in murine Colon-26 and B-16 tumors. Biotechnol Lett 2003; 25: 149-53.

17 Carpentier AF, Chen L, Maltonti F, Delattre JY. Oligodeoxynucleotides containing CpG motifs can induce rejection of a neuroblastoma in mice. Cancer Res 1999; 59: 5429-32.
18 Carpentier AF, Xie J, Mokhtari K, Delattre JY. Successful treatment of intracranial gliomas in rat by oligodeoxynucleotides containing CpG motifs. Clin Cancer Res 2000; 6: 2469-73.

19 Baines J, Celis E. Immune-mediated tumor regression induced by CpG-containing oligodeoxynucleotides. Clin Cancer Res 2003; 9: 2693-700.

20 Buhtoiarov IV, Sonde PM, Eickhoff JC, Rakhmilevich AL. Macrophages are essential for antitumour effects against weakly immunogenic murine tumours induced by class B CpG-oligodeoxynucleotides. Immunology 2007; 120: 412-23.

21 Mason KA, Neal R, Hunter N, Ariga H, Ang K, Milas L. CpG oligodeoxynucleotides are potent enhancers of radio- and chemoresponses of murine tumors. Radiother Oncol 2006; 80: 192-8.

22 Du HY, Xia SY, Song HF, Li N, Shang MM, Zou J, et al. Structureefficacy relationships of immunostimulatory activity of CpG-containing oligodeoxynucleotides on mouse spleen cells. Acta Pharmacol Sin 2007; 28: 1637-44.

23 Walker PS, Scharton-Kersten T, Krieg AM, Love-Homan L, Rowton ED, Udey MC, et al. Immunostimulatory oligodeoxynucleotides promote protective immunity and provide systemic therapy for leishmaniasis via IL-12- and IFN-y-dependent mechanisms. Proc Natl Acad Sci U S A 1999; 96: 6970-5.

24 Harandi AM, Eriksson K, Holmgren J. A protective role of locally administered immunostimulatory $\mathrm{CpG}$ oligodeoxynucleotide in a mouse model of genital herpes infection. J Virol 2003; 77: 953-62.

25 Gramzinski RA, Doolan DL, Sedegah M, Davis HL, Krieg AM, Hoffman SL. Interleukin-12- and gamma interferon-dependent protection against malaria conferred by $\mathrm{CpG}$ oligodeoxynucleotide in mice. Infect Immunol 2001; 69: 1643-9.

26 Blackwell SE, Krieg AM. CpG-A-induced monocyte IFN-gammainducible protein-10 production is regulated by plasmacytoid dendritic cell-derived IFN-alpha. J Immunol 2003; 170: 4061-8.

27 Ballas ZK, Rasmussen WL, Krieg AM. Induction of NK activity in murine and human cells by $\mathrm{CpG}$ motifs in oligodeoxynucleotides and bacterial DNA. J Immunol 1996; 157: 1840-5.

28 Shan BE, Hao JS, Li QX, Taqawa M. Antitumor activity and immune enhancement of murine interleukin-23 expressed in murine colon carcinoma cells. Cell Mol Immunol 2006; 3: 47-52.

$29 \mathrm{Nie} \mathrm{H}$, Meng LZ, Zhang H. Effects of accidental exposure to external cause of gingerol on endotoxemia mouse model induced by heatstroke. Zhongguo Zhong Xi Yi Jie He Za Zhi 2006; 26: 529-32.

30 Serebrisky D, Teper AA, Huang CK, Lee SY, Zhang TF, Schofield BH, et al. CpG oligodeoxynucleotides can reserae $T_{h 2}$-associated allergic airway responses and alter the B7.1/B7.2 expression in a murine model of asthma. J Immunol 2000; 165: 5906-12.

31 Kitagaki K, Jain VV, Businga TR, Hussain I, Kline JN. Immunomodulatory effects of $\mathrm{CpG}$ oligodeoxynucleotides on established $\mathrm{T}_{\mathrm{h} 2}$ responses. Clin Diagn Lab Immunol 2002; 9: 1260-9. 